Cahiers de recherches médiévales

\title{
Quand la fiction se mêle à l'histoire
}

Un combat dans les Faits des Romains

\section{Catherine Croizy-Naquet}

\section{CpenEdition}

Journals

Édition électronique

URL : https://journals.openedition.org/crm/1392

DOI : $10.4000 / \mathrm{crm} .1392$

ISSN : 1955-2424

Éditeur

Honoré Champion

\section{Édition imprimée}

Date de publication : 30 octobre 1998

Pagination : 55-68

ISSN : $1272-9752$

\section{Référence électronique}

Catherine Croizy-Naquet, "Quand la fiction se mêle à l'histoire », Cahiers de recherches médiévales [En ligne], 5 | 1998, mis en ligne le 01 octobre 2007, consulté le 15 décembre 2022. URL : http:// journals.openedition.org/crm/1392 ; DOI : https://doi.org/10.4000/crm.1392

Ce document a été généré automatiquement le 15 décembre 2022.

Tous droits réservés 


\title{
Quand la fiction se mêle à l'histoire
}

\author{
Un combat dans les Faits des Romains
}

\author{
Catherine Croizy-Naquet
}

1 Au début du XIIIe siècle, pour faire une œuvre historique, il n'est point d'autre méthode que la compilation des écrivains du passé ${ }^{1}$. La compilation est à la fois traduction des écrits anciens latins et agencement de ceux-ci, selon un sujet déterminé. L'auteur des Faits des Romains travaille officiellement sur quatre sources latines qu'il prend soin de nommer, la Vie de César de Suétone, la Conjuration de Catilina de Salluste, la Pharsale de Lucain et les Commentaires de la guerre des Gaules de César ${ }^{2}$, sans compter les sources secondaires, plus ou moins officieuses, dont il s'inspire pour nuancer et compléter les données initiales ${ }^{3}$. Son projet était de retracer la vie des douze empereurs de Rome mais, faute de temps ou d'envie, il s'est contenté de celle de Jules César, qui donne déjà lieu à une œuvre d'une importance considérable4. Pour composer son récit, le compilateur use successivement de chacune de ses sources de référence : pour la Guerre Civile, il recourt à Lucain, n'hésitant pas cependant à compléter par l'une ou l'autre de ses autres sources quand il se trouve dépourvu de matière ${ }^{5}$. À manier des sources latines de nature, de facture et de contenu si différents - la prose de César n'a rien de similaire aux vers de Lucain -, il ne pouvait prétendre faire une traduction littérale. Et s'il respecte presque inconditionnellement la trame des événements, comme tout bon historien de l'époque, il doit, dans le même temps, la transposer en langue vernaculaire et en prose, ce qu'il accomplit avec un talent indéniable. La transposition ne s'arrête toutefois pas au stade de la traduction élaborée, elle s'accompagne d'éclairages, de nuances, de suppressions ou à l'inverse d'ajouts qui témoignent d'une influence littéraire et scolaire contemporaine de l'auteur.

2 S'agissant des ajouts, leur nature est variable, allant de l'introduction de dialogues et monologues à l'invention de portraits, le plus remarquable, par leur fréquence, étant la création de toutes pièces de batailles individuelles ou collectives ${ }^{6}$. B. Guenée minimise ces ajouts, considérant l'ampleur de l'œuvre et la fidélité aux sources qui la caractérise le plus souvent ${ }^{7}$. Ils s'imposent pourtant régulièrement ${ }^{8}$. Dans l'épisode de la bataille de Pharsale, pour lequel l'auteur s'inspire en grande partie de Lucain, un certain nombre de combats sortent de l'imagination du compilateur. L'un d'entre eux met en scène les 
exploits et la mort d'un personnage historique, allié de Pompée, Lucius Domitius'. Il est intéressant de voir comment le compilateur combine la vérité historique puisée dans la source et l'invention, autrement dit comment l'histoire et la fiction sont associées pour produire un récit qui se veut et qui se dit historique. En découle une conception de l'histoire propre à l'époque, sinon au compilateur dont l'œuvre est exemplaire d'un genre en voie d'élaboration.

Réécrite d'après Lucain, la bataille de Pharsale est l'objet d'un développement fort conséquent dans les Faits des Romains, le compilateur relatant dans le plus grand détail les différents événements, mêlant les scènes de préparatifs, les tergiversations de Pompée, les annonces prophétiques et, surtout, les batailles individuelles ou collectives $^{10}$. Lorsqu'il ajoute l'épisode consacré à Domitius, il ne fait qu'opérer une greffe à la trame préexistante fournie par Lucain $^{11}$ : à ce moment du texte, il est question du personnage de Domitius. L'allié de Pompée n'est pas un inconnu, l'auteur l'ayant déjà présenté auparavant, à l'instar de ses sources. Domitius est un ancien consul et un ancien prêteur de Rome; c'est l'un des ardents partisans de Pompée, le défenseur malheureux de Corfinium où il a été vaincu et livré par ses hommes à César qui lui laisse la vie sauve, en dépit de son souhait de mourir :

Cesar s'aperçut bien que cil voloit lues morir, plus que vivre par son otroi et par son

don; car desdaign sambloit a Luce Domice se il vivoit par la merci Cesar. (p. 376,

1. 23-25)

Domitius est donc un personnage qui prend une part active à la vie de Rome. S'il est de second plan, il est néanmoins emblématique des figures qui gravitent autour de Pompée et de Caton. C'est un acteur historique d'une relative importance qui n'est pas le pur produit de l'imagination du compilateur.

Dans ce passage, l'invention consiste en l'ajout d'un combat. L'auteur néglige en effet les réflexions de Lucain pour leur substituer une amplificatio ${ }^{12}$ qui, par sa nature, se fond dans l'ensemble des différentes batailles de Pharsale. L'originalité est de focaliser le regard sur Domitius et d'en faire le seul véritable héros au cours de ce qui s'apparente à une scène filmée. Loin d'être une greffe inexplicable, l'ajout de ce combat se justifie et se légitime par le contexte général de la bataille dont il constitue l'une des péripéties. Il entre dans la logique d'un récit qui retrace un affrontement meurtrier et dans la logique du texte tout entier où il est question, en bref, de la conquête du pouvoir, en partie par la force.

6 L'insertion de la description du combat s'opère d'ailleurs sans rupture, comme le montrent les modalités d'introduction. Le compilateur use du terme merveille, pour désigner la conduite héroïque de Domitius :

De Domice avint il iluec une merveille. (p. 531, 1.32)

7 Le substantif fait transition avec ce qui précède et permet d'entrer de plain-pied dans la digression, sans pour autant briser l'harmonie narrative pré-établie. Il souligne en outre l'importance de l'épisode, se faisant captatio benevolentia du lecteur/auditeur. Il est surtout une manière de qualifier la prouesse de Domitius, le véritable héros - sinon le seul - de l'épisode; la place du nom propre en tête de phrase est, à ce titre, éloquente. En même temps, le terme merveille est un indice métatextuel, c'est-à-dire le signalement d'un décrochage par rapport à la source, la désignation explicite de ce qui est greffé sur la trame historique. N'est-ce pas une manière de dire que la fiction entre momentanément dans l'histoire? 
8 Le système conclusif s'inscrit avec la même aisance dans le récit. Le combat se clôt sur la défaite de Domitius, vaincu par le plus grand nombre :

Ançois que il poïst avoir secors de Pompee ne dou Senat [...], il ot tant dou sanc

perdu par les plaies, dont il ot meintes, que il li covint a chaoir. (p. 534, 1. 9-13)

9 Le compilateur utilise à nouveau Lucain, tout en ajoutant quelques détails; il relate l'arrivée de César, ses propos, puis le discours de Domitius qu'il amplifie et enfin la mort $\mathrm{du}$ héros $^{13}$. Ainsi inséré, l'épisode du combat ne bouleverse nullement l'enchaînement narratif, logique et chronologique, le compilateur pouvant faire illusion auprès d'un public non averti, ignorant de Lucain. À la merveille de Domitius se superpose celle du compilateur qui se révèle maître en matière de manipulation.

Fondu dans la trame d'ensemble, l'épisode constitue cependant un espace clos, à lire comme une péripétie issue de la Pharsale de Lucain et comme un épisode de la vie de Domitius. Le passage fonctionne de manière autonome, concentré sur la figure de Domitius qui est au centre de l'action. Il est construit en fonction des actes du héros, qui ne sont que des combats successifs, et des réactions qu'ils suscitent. Tout se passe comme si le projecteur ne le quittait pas un seul instant, enchainnant plan sur plan, à la faveur d'un rythme volontairement trépidant que suscite la suite de phrases courtes :

Il [Antoine] broche vers Domice. Tel cop li done parmi li chief, que tot li fent l'hiaume. (p. 532, 1. 24-25)

Le compilateur alterne volontiers tous les ressorts du récit: de brèves descriptions restituent avec précision la scène et le déroulement des combats :

il li trencha la nasiere dou hialme et li embati le brant en la face au travers dou nes.

(p. 532, 1. 28-29)

La narration repose sur la succession de combats singuliers, d'homme à homme, puis collectifs, un contre tous :

Antoines et Lelius et autres connestables se vertirent tuit a lui [Domitius]. (p. 532,

1. 2-4)

Des monologues sont introduits, qui s'imposent comme des commentaires en direct et à chaud et comme une intériorisation de l'événement, et donnent donc accès à une conscience :

«0, dist-il, quant einsi est que morir doi, ce me doit reconforter...» (p. 533, 1. 25-26)

Seul Domitius a droit à la parole, ce qui prouve que, selon le souhait du compilateur, il est le seul véritable héros du passage et que la bataille est perçue à travers ce qu'il en ressent. Axées sur les actes du héros ou orientées par le regard qu'il porte sur sa situation et sur ses adversaires, les données de l'événement ne sont donc que partiellement objectives. Par sa complétude, le texte créé se présente comme un morceau détachable, un îlot de création et d'imagination qui n'en reflète pas moins les enjeux de l'œuvre entière. A cet égard, il est un microcosme à l'image du macrocosme qu'est l'œuvre fondée, pour une grande part, sur le récit de batailles dont celle-ci n'est qu'une variante.

Pour créer et nourrir cet épisode, le compilateur ne peut disposer de sa seule source ; aussi fait-il appel à un arrière-plan littéraire et culturel qui lui est propre, en recourant en particulier aux chansons de geste. Jadis, P. Meyer a constaté que tout l'épisode issu de la Pharsale était écrit en style épique, au point d'émettre l'hypothèse, aussitôt infirmée, que l'auteur avait peut-être sous les yeux une chanson de geste inconnue consacrée à César ${ }^{14}$. Hypothèse séduisante dans la mesure où les récits de bataille sont 
imprégnés du modèle épique et du modèle romanesque, le second se faisant l'écho du premier ${ }^{15}$. Dans l'épisode qui met Domitius en première ligne, l'influence épique est sensible en plusieurs points, d'abord dans la structuration du récit. Certes, l'absence de laisses, dont le rôle est fondamental quant au fonctionnement du récit ${ }^{16}$, ne rend pas évidente a priori la marque ou l'empreinte épique caractérisant le texte historique en prose. Néanmoins, l'épisode offre ici quelques-unes des ressources inhérentes au jeu d'enchaînement des laisses, faisant alterner passages destinés à la progression du récit, passages plus brefs où le monologue intérieur laisse affleurer l'inquiétude, la ténacité, voire le pathétique ${ }^{17}$. La technique de la reprise partielle participe également de l'écriture épique ${ }^{18}$. D'un combat à l'autre, il s'opère de menus changements, le héros combattant plusieurs adversaires, puis un seul, puis plusieurs ${ }^{19}$. Mais le déroulement proprement dit des combats, leur facture restent quasiment similaires, hormis une légère progression faite de subtils déplacements qui permettent au récit d'aboutir à la mort annoncée et programmée de Domitius :

Et l'en li lançoit et treoit, et fu tant feruz, que il ot mout de plaies ou cors et mout ot dou sanc perdu. (p. 534, l. 5-6)

L'influence épique est plus remarquable encore dans l'usage des motifs choisis ${ }^{20}$. Aux chansons de geste, le compilateur emprunte tout ce qui ressortit au motif narratif de la bataille, dans la terminologie de J.-P. Martin ${ }^{21}$, dont la mobilisation des forces :

[...] avint que Domice fu avironnez et assailluz de moutes parz... (p. 532, 1. 1-2),

l'armement évoqué tout au long des combats par pièces, avec une mention de l'enseigne, signe de ralliement ou arme :

Lelius tint l'ensaigne atot l'aigle d'or. Il en feri Domice en l'escu, mes il nel pot movoir des arçons. (p. 532, 1. 9-10),

les combats singuliers :

Il feri a cel assaut Gaddiel [...]. Puis feri Pyrum, un vaillant chevalier [...] (p. 533,

1.12-116),

ou les mêlées - encore n'y a-t-il pas de mêlées au sens propre puisque Domitius est seul contre tous $^{22}$. Le compilateur prend plaisir à manipuler le jeu d'opposition entre un seul et tous les autres, ce que souligne l'usage de termes collectifs comme estors (p. 532, 1. 6), ost (p.532, 1.4), par contraste avec le nom de Domitius ou le pronom personnel tonique :

Il en ocioit et abatoit tant, que li tas en estoit hauz entor lui. (p. 532, 1. 8-9)

Le pronom sujet il qui débute la phrase trouve lui en écho pour la clore, comme si la structure même reproduisait la supériorité momentanée de Domitius, dominant et enfermant ses adversaires en tas. Cependant, à la différence du héros de la chanson de geste, le personnage n'affronte pas seul un grand nombre de Sarrasins; le trait se veut plus réaliste ${ }^{23}$ :

Il en versa plus de quatorze... (p. 534, 1.3)

Lors des attaques, le motif privilégié reste celui du combat à l'épée ${ }^{24}$ :

Lors empoigna l'espee, si conmença a ferir a deus meins. Cui il consuivroit a cop, ses

termes estoit feniz. (p. 533, 1.11-12)

22 À l'issue de l'affrontement qui coïncide avec la mort de Domitius, le compilateur introduit un bref planctus prononcé par Pompée sur la dépouille de son allié, planctus absent de la source. C'est un motif récurrent dans la chanson de geste, rhétorique et lyrique selon J.-P. Martin ${ }^{25}$ : 
«Ahi, dist il, bons chevaliers loiax, discrez et sages en conseill, seürs et fiers en estor; tant fetes a pleindre. Se Rome eüst auques de tex deffendeors come vos estiez, ele poïst longuement sa franchise garder et meintenir.» (p. 535, 1. 15-18)

clichés existants, nuancés ou remaniés ${ }^{30}$ :

Antoines et li soen ferirent tant sor son escu que il li despecierent tot... (p. 533,

$$
\text { 1. 5-6) }
$$
témoigne d'une entrée de la fiction dans l'histoire, ou plutôt qui affiche le recours à la fiction pour écrire l'histoire. Pourquoi le compilateur nourrit-il l'histoire de fiction? Pourquoi à la compilation de sources anciennes, authentiques et suffisantes, de sources qui font autorité ${ }^{31}$, éprouve-t-il le besoin d'ajouter la compilation d'œuvres littéraires contemporaines, ou d'introduire des fragments adaptés de textes contemporains?

La méthode historique adoptée laisse deviner une préoccupation qui, par ailleurs, n'est jamais avouée au cours de l'œuvre, celle de sa réception. Sans aller jusqu'à évoquer la notion de divertissement, absente des Faits des Romains ${ }^{32}$, la propension du compilateur à transposer parfois le texte en s'aidant des textes littéraires induit une conception 
singulière du mode de transmission de l'histoire. Il s'agirait de montrer, de faire voir l'événement et partant, de le faire revivre, la fiction n'étant que le ressort d'une démonstration historique. Cette ambition du compilateur peut expliquer les effets de dramatisation présents tout au long de ce texte, qui proviennent de l'adoption de procédés rhétoriques. L'écriture qui s'élabore est une écriture de l'ostentation qui vise à suggérer le tragique de la situation, grâce au travail sur le rythme surtout. Celui-ci est d'abord fondé sur une succession de phrases brèves et longues qui semblent épouser au plus près les étapes de la bataille. Tout se passe comme si la prose se déployait ou se rétractait pour restituer les mouvements du combat et les échanges entre les adversaires, et pour reproduire leur vivacité :

quar il voit bien que tant com cil soit en estant, n'avra Cesar victoire. Il broche vers Domice. Tel cop li done parmi le chief que tot li fent l'hiaume. Ne fust la coiffe, qui tant ert treslice et serree, il l'eüst maumis a celui cop. Lors li gita Antoines un cop de retrete et le feri en mi le vis, si que il li trencha la naisere dou hialme et li embati le brant en la face au travers dou nes. Domices, qui se senti navré, cuida le sens changier. Lors hauce l'espee, si quida ferir Antoines et soi vengier. (p. 532, 1. 23-30)

En allant d'une phrase courte à une phrase de plus en plus longue, l'auteur crée un effet d'élargissement, de l'attaque à la réplique, du geste à sa conséquence inévitable, du combat solitaire au duel. Cette pratique constante dans le récit des batailles est confortée par l'usage de reprises ou de répétitions qui permet de scander, voire de marteler la phrase, et de rendre en conséquence la cruauté du combat :

Chascuns li lançoit, chascuns li feroit. (p. 532, 1. 6-7)

Cruauté du reste mise en valeur grâce aux synonymes qui ponctuent le texte dans son entier : occision qui ouvre le passage (p. 531, 1.33) est glosé par destruction (p. 532, 1. 3), puis par masecre (p. 532, 1.5), estors (p. 532, 1.6) et chaple (p. 532, 1. 19). Toute la bataille est l'objet d'une mise en scène travaillée en vue de solliciter l'imagination par une captation des sens, de la vue et de l'ouie, par le biais des comparaisons ${ }^{33}$ et par le choix d'un vocabulaire imagé et précis :

[...] li tas en estoit hauz entor lui. (p. 532, 1. 8-9)

Dans cette scène, la caméra ne quitte pas un seul instant le véritable héros, Domitius. Les antithèses ou le jeu des oppositions qui distinguent Domitius de ses adversaires et montrent en action un héros seul contre tous contribuent à la dramatisation de l'épisode ${ }^{34}$. La mise en scène repose également sur une lente gradation, au moyen de quelques déplacements ténus qui annoncent la mort de Domitius. Le compilateur conduit le lecteur/auditeur de la résistance victorieuse du héros vers sa fin inexorable, suggérée à la fois par les faits -Domitius est acculé de toutes parts et blessé ${ }^{35}$ - et par la pleine conscience que le héros prend de sa situation, rendue plus éloquente par l'évocation des réflexions intérieures et par l'introduction du discours direct:

«Or n'i a dont que de vendre ma mort. » (p. 533, 1. 32)

La dernière touche à la mise en scène tragique est donc l'humanisation produite par le regard du héros sur lui-même, sur sa mort inévitable qui l'incite à se jeter à corps perdu dans la bataille finale. L'accent est mis sur l'espèce de folie meurtrière qui l'anime et l'apothéose sanglante qui en découle, avant que lui-même ne s'éteigne dans le bain de son propre sang:

Ce fu granz dolors. (p. 534, l. 13)

Cette sobre formule qui contraste avec la débauche de violence précédente, plus que tout commentaire, confère tout son tragique à la situation. 

point d'un recours constant aux effets de réel. La fiction est en effet utilisée pour faire vrai, sinon vraisemblable. Le compilateur ne perd jamais de vue la nature de son travail : il fait de l'histoire. Le plus apparent - et ce n'est pas propre à ce passage -, c'est la transposition du combat romain en combat médiéval, celui que décrivent chansons de geste et romans courtois : combat à cheval, combat à l'épée, combat avec toute la panoplie d'armes requises:

L'ensaigne chaï a terre. Lelius la cuida relever, mes Domices le hasta et li dona un tel cop de l'espee, que il li trancha le banc hauberc sor les costes au somet de s'espee, et li fendi la char iiij doie en parfont. (p. 532, 1. 13-16)

L'anachronisme est sans nul doute concertés ${ }^{36}$. Il participe de la volonté de projeter des faits, valeurs et coutumes dans le passé, afin de rendre celui-ci plus immédiat, plus accessible et donc plus réel. Le gommage de la distance temporelle entre dans la démarche générale qui vise à éradiquer toute pétrification des événements dans un lointain définitivement perdu pour le présent. Le réalisme procède ensuite de la crudité des images dans les scènes décrites, trait fréquent dans la littérature médiévale. L'exemple-type est ici l'utilisation, comme arme de combat, d'une partie du corps d'un chevalier tué :

Lors prist Domices le bras Gaddiel le Hongre atote l'espaule et le col et la teste, que

il meïsmes avoit tantost ocis ilec [...] (p. 533, 1. 33-34)

Assaut spectaculaire comme le manifeste la comparaison:

Cui il consuivoit a cop, il l'abatoit contre terre autresi conme se il fust feruz d'un mangonel. (p. 534, l. 1-2)

Ces scènes sont d'autant plus atroces que les protagonistes ne sont pas des chevaliers anonymes, de simples figurants, des marionnettes animées le temps d'un combat. Le compilateur prend soin de les nommer, de noms certes fantaisistes mais qui ont au moins le mérite d'ancrer les combattants dans une famille, dans un groupe social, dussent-ils n'apparaitre qu'une seule fois dans le texte. Il est ainsi question de Gaddiel, un Hongre (p. 533, 1. 13), de Pyrum (p. 533, 1.17), de Dorothee, Merien et Garon (p. 534, 1. 8), l'auteur alternant noms de fantaisie, noms romanisés ou noms grecs ${ }^{37}$, et les mêlant aux noms de personnages historiques, Antoine et Domitius. La combinaison de noms historiques et fictifs permet de donner aux premiers davantage de poids historique, en les situant dans un ensemble d'individus, et aux autres d'accéder, le temps de leur nomination, au rang d'acteurs de l'histoire. Le lecteur/auditeur non averti ne pouvait sans doute guère démêler le vrai du faux en la matière, d'autant que ces inventions concernent, pour l'essentiel, les personnages secondaires. Qu'Antoine ait ou non un neveu, Aurelien, engagé dans la bataille, importe peu aux yeux du compilateur; ce qui est fondamental, c'est de rendre toute la portée dramatique de l'événement.

41 L'invention de cette bataille est une belle illustration de la fusion qui s'opère entre histoire et fiction. Si dramatiser et faire vraisemblable guident le travail du compilateur - ce qui vaut, peu ou prou, pour toute addition à la source initiale -, mettre en valeur la problématique de l'œuvre à travers un personnage est une motivation tout aussi fondamentale. Que cette greffe fictive intervienne au sein de la bataille de Pharsale n'est pas anodin. D'un point de vue quantitatif, l'ampleur textuelle consacrée à l'épisode provenant de la Pharsale est lié à l'enjeu qu'est l'issue de la Guerre civile pour le destin de Rome. En ouvrant la scène sur une appréciation de la bataille de Pharsale :

Cahiers de recherches médiévales, 5 | 1998 
En cele grant occision que l'en fesoit ilec, ou li cors des rois et des senators chaoient mesleement li un sor les autres [...] (p. 531, 1. 34) fidélité à la cause de Pompée, c'est-à-dire à une certaine idée de Rome. Plus que pour un homme, il s'engage pour l'idée qu'il se fait de Rome: ses prises de parole ou la restitution de ses propos par le discours indirect sont toujours habitées par l'idée du sacrifice de soi au nom de la franchise de Rome. Notion récurrente qui guide et qui explique son comportement et dont Pompée prend lui-même acte lors du planctus qu'il prononce sur le corps de Domitius ${ }^{40}$. Le sacrifice de soi à une cause collective n'exclut toutefois pas une humanisation du personnage, à l'instar des héros épiques dépeints dans leurs doutes ou dans leurs souffrances ${ }^{41}$. Domitius est confronté à la peur :

[...] et il fu toz espoentez il se senti a terre et il vit que l'en enpressoit de totes parz ne ne s'avoit de coi deffendre... p. 533, 1. 20-22),

le compilateur prend la mesure d'un événement emblématique de la puissance de Rome et de sa déchéance, et d'un événement fondateur de la puissance de César par la disparition de Pompée. À travers le récit, il convient de suggérer que la défaite de Pompée à l'issue de l'affrontement entre les deux camps n'est pas déshonorante : la présence à ses côtés de combattants de la valeur de Domitius en est la preuve. L'attitude héroïque du personnage a peut-être pour effet de conforter indirectement l'image favorable de Pompée telle qu'elle est présentée tout au long du texte ${ }^{38}$.

À partir d'un personnage historique ${ }^{39}$, le compilateur s'efforce de construire en Domitius un héros épique. Plus exactement, il exploite les éléments historiques qu'il combine à des éléments fictifs pour transformer un personnage historique en héros. La tâche est d'autant plus aisée que Domitius est tué et disparaît du texte, sans contrevenir, au final, à la vérité historique. Par touches successives, le compilateur brosse le portrait d'un héros dont la qualité fondamentale est la qualité guerrière. Au cours des épisodes où il apparaît, Domitius est uniquement présenté dans des scènes de bataille et toujours qualifié du point de vue de sa prouesse :

Mes Lucius Domicius fu pesanz et por les armes et por la grant force dont il estoit, et se tint vertueusement. (p. 375, 1. 27-28)

la rage et au désespoir :

Il escumoit d'angoisse plus que nus vers et se defandoit [...] (p. 533, 1. 3-4)

Il se dépasse lui-même avant de mourir héroïquement, avec résignation et avec une acceptation bienheureuse puisqu'il meurt librement :

«De tant puis estre a aise que je muir frans et que je ne te voi pas seignor de Rome encore [...]. Por ce m'en vois plus liez et plus seürs de cest monde, car la ou je muir sui je en grant esperance que Pompee ait la victoire et que il te face comparer ta desloiauté.» (p. 535, 1. 5-11)

En Domitius, le compilateur campe un type de héros épique, qui place son sens de l'honneur, ses qualités de fidélité inconditionnelle, sa vaillance physique à toute épreuve au service d'une cause dépassant sa personne et ses intérêts. Manque sans doute le dévouement sans bornes à la cause religieuse, mais l'image de l'engagement total s'impose en compensation. La figure héroïque de Domitius est donc tout entière dans ses actes, ses propos en constituant l'extension et la justification.

Intéressante en elle-même, la peinture de Domitius ne prend pourtant tout son sens que confrontée à celle de César. Déjà pressentie et annoncée auparavant, l'opposition entre les deux personnages est ici radicale. À Domitius voué à une cause collective fait 
pendant César qui recherche, dans la Guerre civile, le pouvoir personnel et qui n'est animé que par son orgueil ${ }^{42}$. Et Domitius de le lui rappeler avant de mourir :

«O Cesar, oncor ne voi je pas que tu soies ou point ou tu cuides monter. Li loiers et li guerredons de tes desloiautez que tu as fetes et des traïsons, qui fez entreocirre peres et fix, cosins et parens por monter en honor, si est la seignorie de Rome que tu cuides avoir.» (p. 534, 1. 32-33, p. 535, 1. 1-3)

Derrière le portrait de Domitius se dessine, en filigrane, celui des héros principaux de la guerre civile, Pompée mais aussi Caton pour qui Rome est l'unique raison d'être, pour qui la vertu est le seul mode de comportement honorable ${ }^{43}$. Domitius peut apparaître comme un double simplifié, «stylisé », des deux personnages : de Pompée, il a l'ardeur à vaincre et la ténacité, mais il n'en a assurément pas la profondeur psychologique, les doutes, les sentiments voire la sensibilité ${ }^{44}$; de Caton, il a le sens du sacrifice et de l'engagement absolu, mais il n'en a ni le charisme ni l'austérité. Figure emblématique des partisans de Pompée, il permet aussi de définir et de situer ceux-ci et, par là-même, de souligner la différence entre les deux camps. Les hommes de César ne sont pas montrés à leur avantage, au moins dans ce passage. Ils sont présentés en masse, combattant tous contre un seul. Et quand ils sont individualisés, ils sont en situation d'échec, vaincus par Domitius comme Gaddiel, Pyrum..., ou bien leur bravoure est dégradée par une conduite dépourvue de noblesse. Pour venger son neveu, Antoine n'oublie-t-il pas la notion de combat d'égal à égal ? ${ }^{45}$

Lors ralie ses homes et s'en vienent a Domice. (p. 533, 1. 2-3)

La mise en valeur du personnage de Domitius est ainsi à lire comme une illustration de l'opposition entre deux hommes, Pompée et César, et entre deux camps. L'épisode met en miroir la problématique qui traverse l'œuvre, à savoir l'opposition entre deux conceptions du pouvoir et entre deux types d'individus aptes à diriger. Ce bref épisode se clôt sur la mort de Domitius, qui préfigure tout à la fois celle de Pompée et celle d'une idée ou d'un idéal de Rome. Il affirme aussi la disparition d'un type de héros, le héros épique, et l'avènement d'un autre type, individualiste et sans aucun doute moins prestigieux, incarné par César. Celui-ci prend d'ailleurs acte de cette disparition, dans une ultime adresse à Domitius :

«Ha! Domice! Domice! Or voi je l'ore que il te convient lessier les armes et la conpaignie Pompee, que tu as tant eüe chiere. Des ore mes se combatront li autres sanz toi. » (p. 534, 1. 25-28)

Dans cette séquence, le grand vainqueur est César, le personnage historique qui, à la cause collective, a substitué la cause individuelle, celui qui relègue dans les arcanes du passé une conception univoque et héroïque de la puissance. Au héros tout d'une pièce à la Domitius, personnage statique attaché à ses valeurs et condamné à en mourir, s'oppose un héros pragmatique, une conscience individuelle. Si le héros épique trouve ici une ultime apothéose, il y trouve également sa fin; si le texte illustre la vitalité de la chanson de geste, il signe dans le même temps le caractère suranné de celle-ci ou au moins son aporie. Domitius est un héros du passé, et le texte, nourri de procédés épiques, de le dire. César est un héros moderne, l'acteur historique qui bâtit son empire comme il élabore son histoire. Dans la logique narrative et historique, Domitius ne peut que sortir du récit, tandis que César en demeure le sujet central et le grand bénéficiaire. En faisant du chef romain un héros doté d'une psychologie complexe, en lui donnant les traits d'un héros problématique, l'auteur montre comment il cherche à produire un récit qui dramatise les faits et donne du relief aux personnages historiques. 
L'ajout de l'ultime combat de Domitius n'est pas simple fantaisie de l'auteur. Il participe d'une vision de la guerre civile, en même temps qu'il véhicule une conception particulière de l'histoire. La construction de la bataille et le montage d'un acteur historique en héros épique sont à comprendre dans une perception plus globale du passé et des sources de référence. Loin d'être arbitraires, ils ne font que conforter les données du récit préexistant. Ils montrent que le compilateur ne prétend pas faire de son récit une archéologie du savoir sur le passé, ni proposer une transcription littérale des textes de référence. Attentif à la réception de son œuvre auprès du public - mais de quel public ${ }^{46}-$, il en recherche d'abord la clarté et, pour ce faire, déploie tous les moyens dont il dispose pour la transmettre aisément. L'important n'est pas seulement de connaître avec précision les événements relatés, mais de les percevoir, d'appréhender leur sens réel et les leçons qu'ils peuvent receler, l'œuvre historique étant conçue comme une œuvre de savoir et de sagesse. Or, comment mieux faire appréhender la réalité qu'en adaptant les faits sinon à un public, au moins à la mentalité propre à l'époque de la compilation? Pour être transmise, l'histoire est ainsi nourrie de fiction, et la fiction qui naît de l'arrière-plan littéraire occidental et médiéval est une mise en scène de l'histoire. Elle permet de substituer à l'emphase poétique de Lucain une prose individualisée, se démarquant du modèle initial par son adéquation aux valeurs de l'époque du prosateur, afin de faire advenir une histoire qui soit évocatrice et qui fasse sens. Dans la conscience de l'auteur, il n'y a pas là trahison du passé ni de la source, mais actualisation d'un passé véridique, sous les aspects du vraisemblable. Les frontières entre histoire et fiction sont brouillées, le translateur se voulant et se disant translateur ${ }^{47}$, tout en parachevant son activité d'historien par un travail inavoué de créateur. Ainsi, la littéralité de l'événement est sacrifiée au profit de sa littérarité, plus expressive, plus suggestive et peut-être plus proche du réel passé. L'entreprise est couronnée de succès, puisque le lecteur/auditeur peu averti ne pouvait guère faire le départ entre la fiction et l'histoire.

L'ajout de micro-récits à la trame narrative est une pratique coutumière du compilateur des Faits des Romains. Il s'opère avec harmonie, les modalités d'insertion se caractérisant par une aptitude à se fondre avec naturel à la logique du récit. La seule distorsion que les digressions apportent au regard de la source - et elle est de taille relève de leur nature et de leur facture. Nourries du fonds littéraire contemporain, de l'univers épique surtout, elles contrastent a priori avec le sujet traité. De fait, tel qu'il est représenté dans cet épisode, Domitius apparaît comme un modèle de chevalier épique. Pourtant, le choix de l'auteur ne contrevient en rien à son dessein d'historien. $\mathrm{Au}$ contraire, la fiction est une dimension constitutive de l'histoire, l'une des modalités de traduction, ou plutôt d'adaptation de la matière historique. Elle met l'histoire en valeur, elle permet de montrer, voire d'exhiber son sens profond. En mariant subtilement histoire et fiction, l'auteur propose sa conception de l'histoire comme réécriture des sources du passé à la lumière des œuvres présentes, et sa définition de l'historien comme le savant metteur en scène d'un passé révolu en un passé actualisé, plus présent que jamais, à la fois prétexte et sujet de réflexion. 


\section{NOTES}

1.Voir B. Guenée, «L'historien et la compilation au XIIIe siècle », Journal des savants, 1985, pp. 119-135.

2.Les Faits des Romains, éd. L.-F. Flutre et K. Sneyders de Vogel, 2 vol., Paris-Groninge, 1938. Cf. P. Meyer, «Les premières compilations françaises d'histoire ancienne, I. Les Faits des Romains, II. Histoire ancienne jusqu'à César », Romania, t. 14, 1885, pp. 1-85. Pour Lucain dont s'inspire l'auteur dans la partie consacrée à guerre civile, voir La Guerre civile, (la Pharsale), l'édition et la traduction d'A. Bourgery et de M. Ponchont, Paris, Société « les Belles Lettres », t. 1-2, 1948.

3.Sur la variété des sources utilisées, sources directes et indirectes, et leur identification, se reporter à l'édition de L.-F. Flutre et K. Sneyders de Vogel, t. 2, pp. 22-29.

4.Dans son prologue, le compilateur précisait en effet : «Et comencerons nostre contre principalment a Juille Cesar, et le terminerons a Domicien, qui fu li douziemes empereres...» (p. 2, 1. 24-26).

5.S'agissant de l'auteur des Commentaires, le compilateur mentionne non César luimême, mais Julius Celsus Constantinus à qui l'œuvre fut couramment attribuée au Moyen Age ; cf. éd. L.-F. Flutre et K. Sneyders de Vogel, t. 2, p. 26-28. Lorsque l'une des sources lui fait défaut pour poursuivre son récit, l'auteur en utilise une autre, comme il l'indique lui-même ; sur cette méthode de travail, voir un passage intéressant, p. 651 et ss.

6.Le compilateur crée par exemple des discours de Caton et de César (p. 674-675), ou bien il invente des descriptions, la plus éloquente étant celle consacrée à Cléopâtre, dépeinte en héroïne médiévale (pp. 626-627) ; cf. notre article, « De quelques figures féminines dans les Faits des Romains ", Cahiers de Recherches Médiévales (XIII -XVe), Varia n 2, 1996, pp. 201-220.

7.B. Guenée, «La culture historique des nobles : le succès des Faits des Romains (XIVeXVe siècles) ", La noblesse au Moyen Âge. Essais à la mémoire de Robert Boutruche, réunis par Ph. Contamine, Paris, PUF, 1976, p. 266.

8.Cf. les notes et les commentaires dans l'édition L.-F. Flutre et K. Sneyders de Vogel, t. 2, pp. 61-223.

9.Le personnage apparaît à deux reprises au cours du récit, avant la bataille de Pharsale. Il est d'abord mentionné comme consul (p. 60, 1. 27; p. 373, 1. 32), et il est présenté en tant que partisan de Pompée et défenseur de Corfinium, épisode au cours duquel il joue un rôle de premier plan comme opposant direct à César (p. 373 et ss.).

10.Faits des Romains, pp. 494-544.

11.Cf. Faits des Romains, pp. 531-534 ; Lucain, op. cit., VII, 597-604.

12.Lucain, op. cit., vv. 600-603. Pour la définition de ce terme, consulter Les arts poétiques du XIIe et du XIII e siècle, Paris, $1^{\text {re }}$ éd., 1923, p. 74 et ss.

13.Faits des Romains, pp. 534-535.

14.Cf. P. Meyer, art. cit., p. 17, p. 29.

15.Lors des récits de bataille s'observent en effet dans les romans maints emprunts ou maintes réminiscences du modèle épique, dans les romans antiques par exemple, dont 
Le Roman de Thèbes; $\mathrm{cf}$. A. Petit, Naissances du roman. Les techniques littéraires dans les romans antiques au XII siècle, Paris, Champion, 1985, vol. 1, pp. 251-329.

16.Consulter D. Boutet, La chanson de geste, Paris, PUF écriture, 1993, p. 159 et ss. 17.Cf. D. Boutet, op. cit., p. 174.

18. Voir D. Boutet, op. cit., p. 175.

19.Voir les Faits des Romains, par exemple, p. 532, 1. 19.

20.Sur les sens à donner à ce terme et la classification à laquelle il se prête, voir J.P. Martin, Les motifs dans la chanson de geste. Définition et Utilisation (Discours de l'épopée médiévale), Centre d'Études Médiévales et Dialectales, Université de Lille-III, 1992.

21.Cf. J.-P. Martin, op. cit., p. 354, p. 368.

22.Faits des Romains, p. 532, 1. 8-9.

23. Voir par exemple Aliscans, Paris, Champion, C.F.M.A., 1990, 2 t. ; traduction B. Guidot, J. Subrenat, Paris, Champion Traductions, 1993 ; aux v. 63 et ss., dix mille Sarrasins surgissent devant Vivien qui prend peur.

24.Cf. J.-P. Martin, op. cit., p. 362, p. 368, qui le définit comme un motif rhétorique. 25.Sur le planctus, consulter P. Zumthor, «Étude typologique des planctus contenus dans la Chanson de Roland ", La Technique littéraire des chansons de geste, Actes du colloque de Liège, Bibliothèque de la Faculté de Philosophie et Lettres de l'Université de Liège, Paris, les Belles Lettres, pp. 219-255 ; «Les planctus épiques », Romania, 84, 1963, pp. 61-69. J.-P. Martin, op. cit., p. 188 et ss, p. 376.

26.Cf. Aliscans, la mort de Vivien, v. 940 et ss.

27. On observe aussi l'importance des liens de cousinages ; cf. le relevé proposé dans l'éd. L.-F. Flutre, K. Sneyders de Vogel, t. 2, p. 31.

28.L'importance du lignage est récurrente dans le texte, qu'elle concerne les personnages principaux comme César et Pompée, ou bien les personnages secondaires ou ceux qui n'interviennent que ponctuellement comme Drappés Brenno (p. 316-317). 29.Faits des Romains, par exemple p. 532, 1. 15-16. Sur les vers et fragments de vers, cf. K. Sneyders de Vogel, "Les vers dans les Faits des Romains ", Mélanges Salverda de Grave, 1933, pp. 293-305 et in Neophilologus, XIX, 1934, pp. 172-180.

30.Cf. J.-P. Martin, op. cit., p. 184 et ss.

31.Sur les sources de référence et leur poids comme autorité, consulter B. Guenée, Histoire et culture historique dans l'Occident médiéval, Paris, Aubier, collection historique, 1980 , p. 131 et ss.

32.Le compilateur n'en souffle mot, à l'encontre de son prédécesseur immédiat de l'Histoire ancienne jusqu'à César qui, dans le prologue, mêle les objectifs de divertir et d'apprendre ; voir éd. de M. Coker Joslin, The Heard Word : A moralized History. The Genesis section of the Histoire ancienne in a text from Saint-Jean d'Acre, University Miss., Romance Monographs, 45, 1986, vv. 251-256.

33.Faits des Romains, p. 532, 1. 6-7.

34.Faits des Romains, p. 532, 1. 2-6.

35.Faits des Romains, p. 533, 1. 19-20.

36.Sur l'anachronisme, voir M. Bakhtine, Esthétique et théorie du roman, trad. du russe par D. Olivier, Paris, Gallimard, 1978, pp. 293-297.

37.L'énumération est plus spectaculaire encore dans les pages qui suivent, où l'auteur aligne à la suite une dizaine de noms propres ; cf. p. 536, [45]. Ce goût pour

l'énumération de noms propres n'est pas propre au compilateur ; il apparaît déjà dans 
le Roman de Brut, de Wace, éd. I. Arnold, Paris, S.A.T.F., 2 t., 1938-1940, par exemple aux vv. 2292-2267 avec les noms des rois, princes et seigneurs de l'armée romaine où se mêlent païens et chrétiens. L'auteur des Faits des Romains aurait-il subi quelque influence dans l'invention des noms propres?

38.Voir l'analyse proposée par G. Spiegel qui met en valeur l'opposition entre César et Pompée qui se double d'une opposition entre César et Caton, César étant toujours la figure du mal ; Romancing the Past : the Rise of Vernacular Prose Historiography in Thirteenth Century France, Berkeley et Los Angeles, 1993, p. 159 et ss. Cette analyse diverge de celle de J. M. A. Beer, A Medieval Caesar, Études de Philologie et d'Histoire, 30, Geneva, 1976, qui voit en César une figure surtout positive.

39.Dans les épisodes où il intervient, le compilateur apporte quelques détails sur le personnage ; cf. par exemple, p. 373 et ss.

40.Faits des Romains, p. 535, 1. 16-18.

41.On peut à nouveau citer Vivien dans Aliscans, qui est en proie à la peur devant l'arrivée de milliers de Sarrasins, vv. 87-95.

42.L'orgueil qui est, comme le souligne J. Le Goff, « le péché féodal par excellence »; cf. Saint Louis, Paris, Gallimard, NRF, 1996, p. 422.

43.Sous la forme substantivale ou adjectivale, le terme apparaît plusieurs fois au cours de la bataille: p. 533, 1. 27 ; p. 533, 1. $32 ;$ p. 535, 1. 5, p. 535, 1. 18.

44.À la différence de Domitius, Pompée n'est pas un modèle de héros épique ; personnage complexe et torturé, partagé en particulier entre le devoir et l'amour, il se définit plutôt comme un personnage de roman.

45.Sur ce point, cf. G. Spiegel, op. cit., p. 165 et ss.

46.Selon B. Guenée, art. cit., il s'agit d'abord d'un public composé d'universitaires, p. 265 et ss.

47.Il prend soin de signaler fréquemment la référence à la source, sous la forme «Lucans dist que.../ce dist Lucans »; exemple, p. 537, 1. 26. Il convient de noter toutefois que dans ce passage inventé, il n'est pas une seule fois fait référence à la source. Est-ce un aveu indirect d'une infidélité momentanée?

\section{AUTEUR}

\section{CATHERINE CROIZY-NAQUET}

Université Paris-III Sorbonne nouvelle 